\title{
Towards measures of the eradicability of rain-splashed crop diseases
}

\author{
$\underline{\text { J. C. Bennett }}^{\text {abe }}$, A. Diggle ${ }^{\text {ce }}$, F. Evans ${ }^{\text {ce }}$ and M. Renton bde \\ ${ }^{a}$ The Satellite Positioning for Atmosphere, Climate and Environment (SPACE) Research Centre, School of \\ Mathematical and Geospatial Sciences, RMIT University, Melbourne, Victoria \\ ${ }^{b}$ School of Plant Biology, The University of Western Australia, Western Australia \\ ${ }^{c}$ Department of Agriculture and Food Western Australia (DAFWA), Floreat, Western Australia \\ ${ }^{d}$ CSIRO Ecosystem Sciences, Floreat, Western Australia \\ ${ }^{e}$ Cooperative Research Centre for National Plant Biosecurity, Canberra, Australian Capital Territory \\ Email: james.cameron.bennett@ rmit.edu.au
}

\begin{abstract}
Controlling rain-splashed crop diseases is an extremely difficult task. Their spread is a complicated process and large-scale field surveys to determine the extent of an incursion over a large area are often economically intractable. A failed attempt at control or eradication of a pathogen can be very costly. In 1996 there was a major incursion of lupin anthracnose in Western Australia, which crippled the albus lupin industry. At the time of the outbreak a wide-spread survey was undertaken to estimate the extent of the incursion. A containment protocol involving broad-scale crop destruction was put into place with the view of eradicating the disease. This eradication attempt subsequently failed due to wild lupins acting as a reservoir for the disease from road verges and non-arable land outside the cropping area. There was also evidence of long distance dispersal vectors such as native budworm. Had all the relevant information related to spread and spatial habitat suitability been collected and taken into account, the decision to destroy the crops may not have been made and significant economic losses to growers may have been avoided. Estimates of the current extent of an incursion based solely on incomplete empirical data are likely to be inaccurate, as are predictions of the future trajectory of an incursion that do not take into account all available information. Therefore any control or eradication attempt based on these estimates and predictions may be ineffective.
\end{abstract}

Simulation modelling is an important method for making the best use of all available empirical data and integrating all available knowledge to predict the spread of rain-splashed crop diseases. With this prediction, an evaluation of the potential success of control or eradication measures may be estimated. This study describes a model that was built to simulate a situation analogous to that of the 1996 lupin anthracnose incursion in Western Australia, for the purpose of identifying general indicators of the eradicability of rainsplashed crop diseases.

We extended the spatiotemporal model AnthracnoseTracer to simulate the spread of lupin anthracnose in a heterogeneous paddock environment analogous to the 1996 conditions. Three control methods aimed at eradication were investigated. A simple detection model was assumed, where the probability of detecting the disease is dependent on the level of passive surveillance and the detectability of the disease.

As part of the preliminary analysis contained in this paper we investigated two scenarios to identify potential indicators of eradicability, based on the time taken to detect the disease. Our preliminary results indicate that rain-splashed pathogens are extremely difficult to eradicate and the chance of successful eradication appears strongly dependent on the level of surveillance of the susceptible areas and the detectability of the disease. The level of surveillance and detectability of the disease may both serve as general indicators of eradicability for rain-splashed crop diseases. We discuss further modelling analyses to be carried out to refine these indicators.

Keywords: Simulation modelling, rain-splashed crop diseases, eradication, lupin anthracnose 


\section{INTRODUCTION}

Rain-splashed pathogens have the ability to spread aggressively, particularly when mediated by highly variable mechanisms such as wind, and are capable even of intercontinental spread (Brown and Hovmøller, 2002). Depending on the environmental conditions, the disease may be capable of long distance jumps and satellite infestations may occur, leading to a patchy population structure. This makes predicting the extent of an incursion a very difficult task.

Simulation modelling is a powerful tool to aid decision makers in the event of a new incursion. Simulations of disease spread and potential responses may be rapidly carried out and the likelihood of successful eradication estimated. Using simulation modelling we can investigate a wide range of environmental scenarios which would otherwise be difficult or impossible to carry out experimentally. Determining the extent of an incursion empirically is an expensive process and is not guaranteed to be accurate.

Rare long distance jumps are often modelled using a fat-tailed dispersal kernel and in these cases the rate of spread no longer approaches a constant rate; but accelerates (Kot et al., 1996). If an incursion of this sort is not detected swiftly and action taken promptly, then the disease may rapidly reach ineradicable proportions.

In this paper we investigated the eradicability of rain-splashed crop diseases by simulating a situation analogous to that of the 1996 lupin anthracnose incursion in Western Australia which devastated the albus lupin industry (Clements et al., 2005). Lupin anthracnose is a devastating disease of lupins. It is caused by the fungus Colletotrichum lupini (Nirenberg et al., 2002), previously known as Colletotrichum gloeosporioides (Paulitz et al., 1995; Sweetingham et al., 1995) or Colletotrichum acutatum (Talhinhas et al., 2002). Lupins are a multi-million dollar commodity in Australia (Australian Bureau of Statistics) and much is known, about the progression of lupin anthracnose epidemics (Shivas et al., 1998; Yang and Sweetingham, 1998; Diggle et al., 2002; Thomas and Sweetingham, 2004; Adhikari et al., 2009, among others). In the 1996 case, after a wide-spread survey following the initial discovery the disease was detected on 133 properties, 128 of which were in the Western Australian northern agricultural region (Sweetingham and Shea, 1998). An eradication attempt involving the large-scale destruction of lupin crops was made. In 1997 it was deemed that the eradication attempt had failed due to wild lupins outside the cropped areas acting as a reservoir for the disease. There was also evidence of long distance spread by the native budworm (Sweetingham and Shea, 1998). This case study highlights the necessity of integrating all possible information regarding the disease and the environment it is found in to guide eradication decisions.

In this paper, we describe how we extended the spatiotemporal model AnthracnoseTracer (Diggle et al., 2002) to simulate spread though a heterogeneous landscape, A heterogeneous paddock map, created from a satellite image, was used to segregate the mapped area into different land uses, namely: lupin crop, verges and other non-arable land where wild lupins may be present, and areas without any lupins. The advantage of the spatial extension is that we can obtain an indicator of the efficacy of surveillance and eradication at regional scales. We also identify two potential candidates to be used as general indicators of eradicability for rain-splashed crop diseases and discuss planned ongoing modelling studies to refine these indicators.

\section{THE MODEL}

\subsection{Original AnthracnoseTracer}

We extended the model AnthracnoseTracer (Diggle et al., 2002), a stochastic model of lupin anthracnose spread over a homogeneous paddock. AnthracnoseTracer adopts a grid-based modelling approach and simulates intra-cell crop growth as the temperature dependent development of growing points including the reduction due to disease. The initial incursion occurs by the sowing of infected seed. Disease development into the formation of potentially infective anthracnose spores is modelled in a cell using a simple temperature dependent latent phase (delay function). When this threshold is passed, the spread of spores, which occurs both within and between cells, is triggered once a rainfall threshold of $2 \mathrm{~mm}$ in a day or $0.1 \mathrm{~mm}$ in an hour is reached. Dispersal of spores is simulated individually in each of these wet hours up to a threshold after which their dispersal is simulated in groups (or clouds). Both rain-splash and wind dispersal mechanisms are simulated. The direction of travel for a spore (or spores) during rain splash is assumed equally likely in all directions, whilst the direction of spores travelling by wind was determined using a normal distribution based on the average wind direction and the standard deviation of wind direction during the wet hour. Half Cauchy distributions determined the distances spores disperse, by either rain splash or wind vectors. The shape parameter for the wind distribution is multiplied by the average wind speed in the wet hour. Both half Cauchy distributions in AnthracnoseTracer were parameterised using field observations. The position after 
dispersal is determined by the vector sum of the rain splash vector and the wind vector. The deposition of a spore on a susceptible lupin growing point is determined using the probability:

$$
S_{p}=1-\exp \left(-k P_{s} T\right)
$$

where $S_{p}$ is the spore deposition probability, $P_{s}$ is the number of susceptible growing points and $T$ is the time of susceptibility, and $k$ is the shape parameter which controls the rate of decrease in the probability of deposition as the number of susceptible growing points by susceptibility time approaches zero $\left(P_{S} T \rightarrow 0\right)$. Equation (1) is commonly used to approximate the proportion of incoming radiation intercepted by a crop canopy (van Heemst, 1986; Diggle et al., 2002). Whether a spore successfully infects the growing point is determined stochastically. The AnthracnoseTracer model was parameterised for the narrow leaf lupin varieties Wonga and Myallie as well as the susceptible Kiev Mutant (albus lupin) variety. In our model we assumed two crop types, Myallie and Kiev Mutant, and the different crops are distinguished in the disease process by the number of potentially infective spores produced per sporulating growing point. For details of the AnthracnoseTracer model the reader is referred to Diggle et al. (2002). We took the original AnthracnoseTracer model in its entirety and made the necessary additions to simulate multiple lupin types as well as spread through a heterogeneous landscape.

\subsection{The heterogeneous paddock map}

We created a map that allowed us to distinguish different paddock uses and also verge areas to simulate the spread of lupin anthracnose through a heterogeneous environment. A satellite image obtained from Google Earth was used to create a suitability map reflecting the differing land uses. The satellite image was segmented into individual paddocks shown in Figure 1. The left hand image is the original satellite image and the image on the right shows the 48 paddocks represented by individual colours. The colours are arbitrary and similar colours do not represent any similarities in environmental conditions or paddock type. The paddock where the initial infection occurs in all simulations is marked by an " $X$ ". The black area in the image on the right in Figure 1 represents the assumed verge areas.
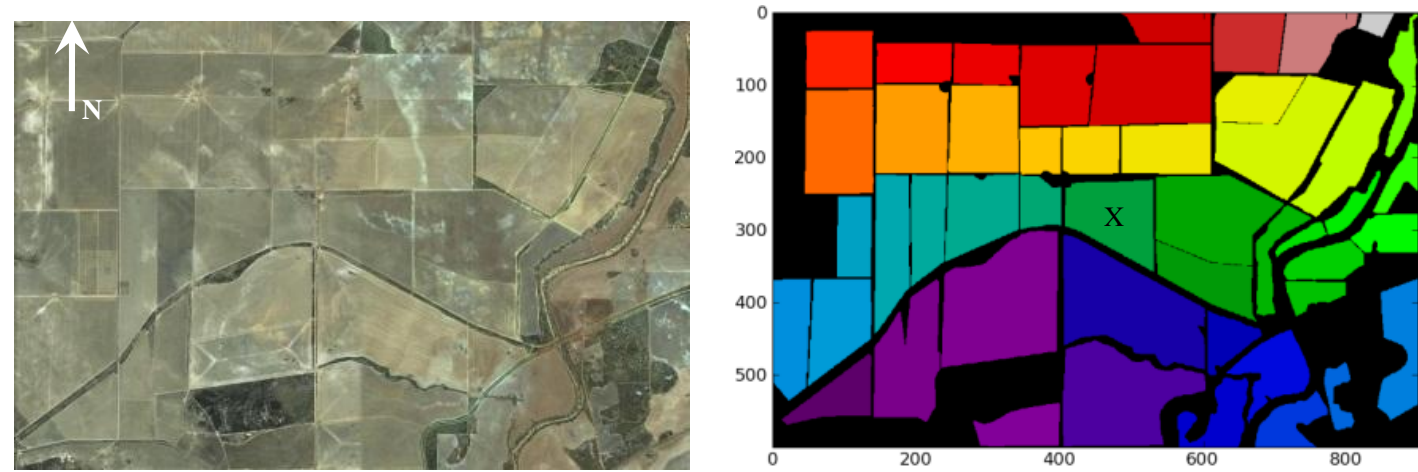

Figure 1. Converting the satellite image into a suitability map. The image on the left shows the original satellite image (ㄷ 2009 Google, (C) 2010 Cnes/Spot Image, Image (C) 2010 DigitalGlobe, (C) 2010 MapData

Sciences Pty Ltd, PSMA) and the image on the right shows the generated suitability map. The " $X$ " in the right image depicts the paddock where the incursion occurs in all simulations. One unit on the suitability map scale corresponds to $10 \mathrm{~m}$

\subsection{Detection}

We assumed a simple detection model where each lupin paddock grid cell has equal probability of being inspected each day and the chance of detecting the disease if present in a cell is governed by an assumed level of detectability. The level of surveillance, $\operatorname{LoS} \in[0,1]$, is the assumed proportion of lupin crop area inspected on each day. The surveillance is assumed to be casual inspection by a farmer which suggests a lower value of $\operatorname{LoS}$ is appropriate. The level of detectability, $\operatorname{LoD} \in(0,1]$, is the proportion of infected growing points in a cell required for a $50 \%$ chance of detection. Therefore $\operatorname{Pr}(D)_{t}$, the probability of detecting the disease at time $t$, is given by

$$
\operatorname{Pr}(D)_{t}=L o S \times \operatorname{Pr}(D I C),
$$

where $\operatorname{Pr}(D I C)$, the probability of detection in a cell, is given by 
Bennett et al., Towards measures of the eradicability of rain-splashed crop diseases

$$
\operatorname{Pr}(D I C)=\frac{I G P}{I G P+L o D \times G P}
$$

where IGP is the number of infected growing points in a cell and GP is the number of growing points in the cell. This formulation allows an estimate of the detectability of a disease to be obtained via a simple estimation of the proportion of infected growing points corresponding to a fifty-fifty chance of detection in a cell. The worst case scenario (corresponding to $L o D=1$ ) in $(3)$ assumes a maximum $\operatorname{Pr}(D I C)$ of 0.5 when all growing points are infected in a cell. The possibility of the disease being more difficult to detect than this is neglected under the assumption that eradication would not be feasible.

\subsection{Eradication measures}

We considered three techniques of control aimed at eradicating the disease. These methods are:

1. Destroy all the lupin in the paddock/s in which the disease was detected, but not wild lupins in verge areas;

2. Destroy all lupin paddocks immediately after detection, but not wild lupins in verge areas;

3. Destroy all lupins within a certain control radius - both crop area and wild lupins in the verge areas.

The assumption was made that control is $100 \%$ effective and there was no remaining disease in the areas where the lupins were destroyed.

\subsection{Dispersal beyond the mapped area}

Anthracnose spores dispersing beyond the mapped area are allocated to a land use which is determined randomly using the proportion of area of each land use in the mapped area as the expected proportion outside the mapped area. Once the land use has been determined, whether the spore infects a susceptible lupin growing point or not is determined by the probability given by equation (1).

\subsection{Model characteristics}

A schematic of the model processes is shown in Figure 2 and the model characteristics assumed to represent the 1996 situation are described in Table 1. All other parameter values are exactly as for the original AnthracnoseTracer model (Diggle et al., 2002). The paddock map is shown in Figure 3. This was created by randomly assigning each paddock (except the paddock where initial infection occurs) of the suitability map

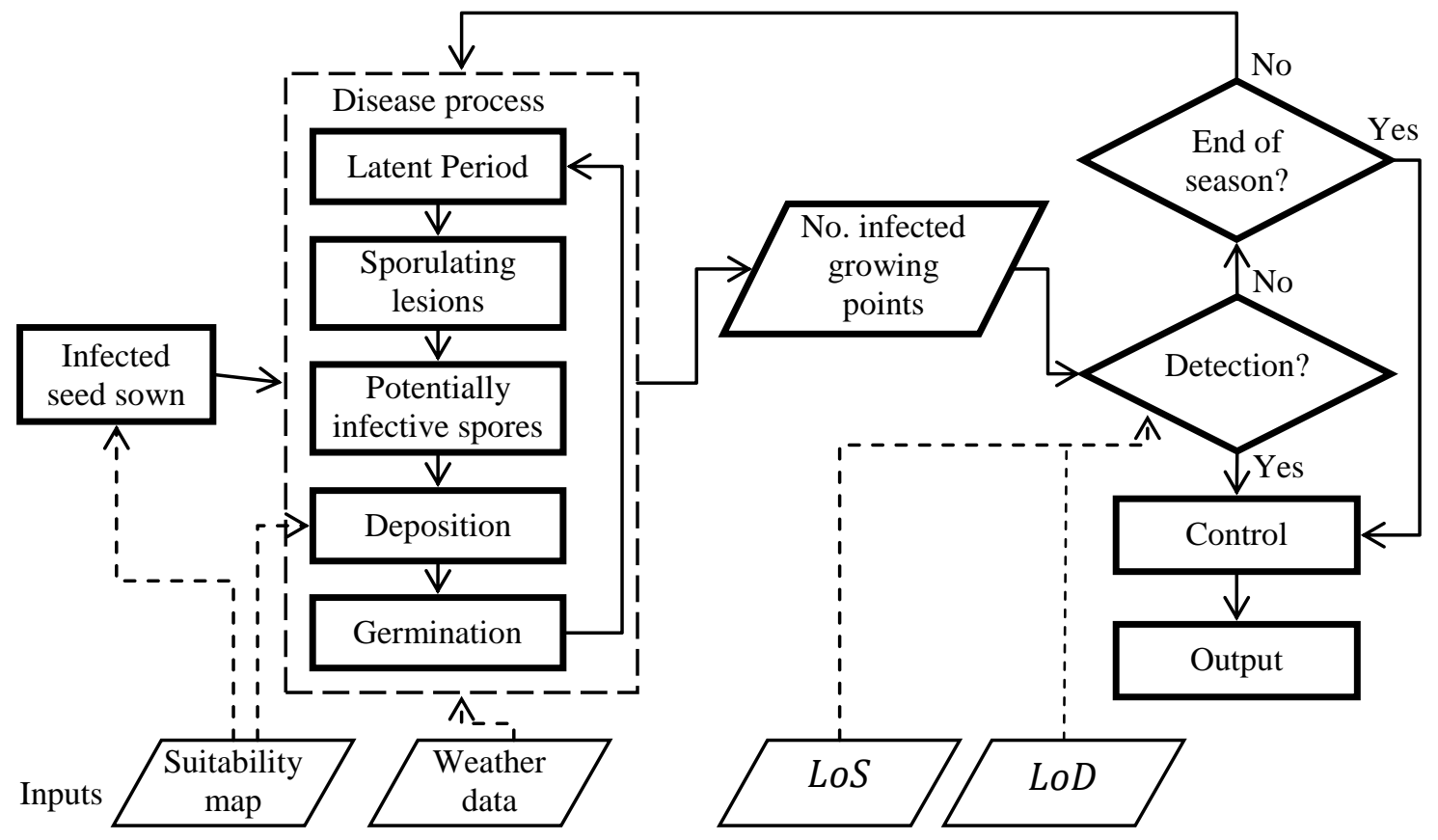

Figure 2. Schematic representation of each model run. Disease progression is simulated until detection occurs or the end of the growing season is reached. 
in Figure 1 with each of the three paddock land uses Kiev Mutant (KM), Myallie (M) and unsuitable (US) according to the assumed abundance contained in Table 1. The lupin abundance is assumed to be the proportion of paddocks for each land use. For the current distribution of $20 \% \mathrm{KM}, 20 \% \mathrm{M}$ and $60 \% \mathrm{US}$ this corresponds to $10 \mathrm{KM}, 10 \mathrm{M}$ and $28 \mathrm{US}$ paddocks. Hence the distribution is not based on the area made up by each land use. We used the hourly weather dataset for Mingenew in 1996 since it is in the Western Australian northern agricultural region where the incursion occurred and there was a good hourly rainfall dataset collected that year. We considered this rainfall dataset to be typical of the scenario of the 1996 incursion. The growing season was assumed to span from the $19^{\text {th }}$ of May to the $8^{\text {th }}$ of November. A random infection point is simulated in the paddock where the initial infection occurs (Figure 1) by selecting a coordinate at random. This is designed to simulate the potential for the point of initial incursion to be close to the paddock boundary resulting in dispersal outside the paddock boundary occurring quicker - thus affecting the control success. The crop type in the paddock where initial infection occurs is assumed to be Kiev Mutant.

\subsection{A more optimistic surveillance and detectability scenario}

In addition to the $L o S=L o D=0.1$ scenario of Table 1 , we investigated the effect of an improved surveillance and detectability scenario on the results of the control methods. We tested the sensitivity of the results by running another scenario with $L o S=0.3$ and $L o D=0.01$ to investigate the change in success in the control methods. This means the amount of crop area inspected per day is $30 \%$ and the proportion of infected growing points in a cell required for a $50 \%$ chance of detection is reduced to $1 \%$.

\section{PRELIMINARY RESULTS}

We performed a number of stochastic model runs for the nominal case presented in Table 1 . We carried out 1000 runs and analysed the chance of eradication for the three control techniques considered.

Successful control for method 1 was achieved in $8.2 \%$ of model runs. A success rate of $18.2 \%$ was achieved for control method 2. For control method 3, an eradication radius of $20 \mathrm{~km}$ resulted in a success rate of $42.4 \%$. Figure 4 shows the cumulative distribution of the maximum distance of infection for each run. The distance describes the radial distance of the circular region where all lupins (paddock and verge) would have to be destroyed (control method 3). For a $95 \%$ probability of success a circular area with radius $400 \mathrm{~km}$ would need to be destroyed. This corresponds to an area of approximately $500,000 \mathrm{~km}^{2}$.

Next we investigated the effect of changing the level of detectability and the level of surveillance from their
Table 1. Model characteristics for the test case assumed to describe the 1996 situation. (KM Kiev Mutant, M - Myallie, US - Unsuitable)

\begin{tabular}{|c|c|}
\hline Parameter & Value \\
\hline$L o S$ & 0.1 \\
\hline$L o D$ & 0.1 \\
\hline Initial $G P$ density $\left(\# / \mathrm{m}^{2}\right)$ & \\
\hline Crop & 40 \\
\hline Verge & $3 \%$ of crop \\
\hline Unsuitable & 0 \\
\hline Lupin crop abundance & $\begin{array}{c}40 \%(20 \% \\
\text { KM, 20\% } \\
\text { M, 60\% US) }\end{array}$ \\
\hline Initial infection crop type & KM \\
\hline $\begin{array}{l}\text { Location of initial infection in } \\
\text { paddock }\end{array}$ & Random \\
\hline $\begin{array}{l}\text { Distribution of lupins in } \\
\text { paddocks surrounding paddock } \\
\text { of initial infection }\end{array}$ & $\begin{array}{l}\text { Scattered } \\
\text { (Figure 3) }\end{array}$ \\
\hline Lupin variety in the verge areas & KM \\
\hline $\begin{array}{l}\text { Rainfall dataset location and } \\
\text { year }\end{array}$ & $\begin{array}{l}\text { Mingenew } \\
1996\end{array}$ \\
\hline Simulation start day & $\begin{array}{c}140 \\
(19 / 05 / 1996)\end{array}$ \\
\hline Simulation end day & $\begin{array}{c}313 \\
(8 / 11 / 1996)\end{array}$ \\
\hline
\end{tabular}

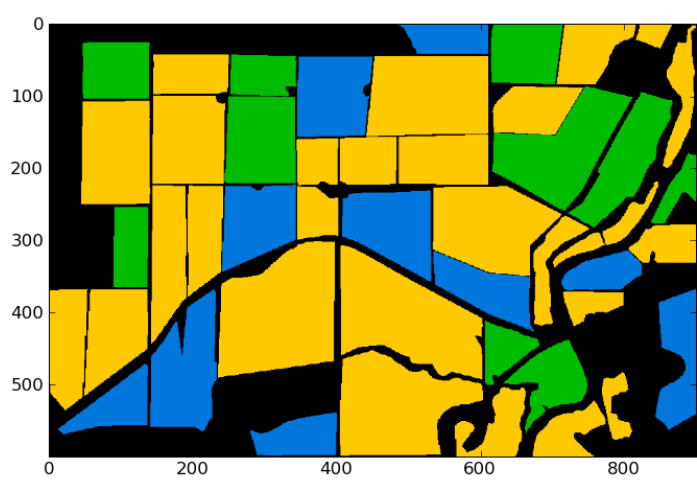

Figure 3. Model paddock map. Blue paddocks correspond to Kiev Mutant (KM), green paddocks are Myallie (M) and yellow paddocks are unsuitable (US). The remaining black area is assumed to be verge. As in Figure 1, one unit on the paddock map scale corresponds to $10 \mathrm{~m}$. 
nominal values and performed 1000 stochastic runs. With the improved scenario described by the parameters $L o S=0.3$ and $L o D=0.01$, representing higher level of surveillance and a greater detectability of the disease, control method 1 was successful $58.8 \%$ of the time and control method 2 was successful $70.9 \%$ of the time. The cumulative distribution for the maximum distance of infection is contained in Figure 5. For a $95 \%$ probability of success for control method 3 a circular area with radius $85 \mathrm{~km}$ would need to be destroyed. This corresponds to an area of approximately $23,000 \mathrm{~km}^{2}$, much less than the original scenario.

\section{DISCUSSION AND CONCLUSIONS}

For the case presented in Table 1 we saw that neither of the paddock control methods (control methods 1 and 2) were reliably effective at eradicating the disease. For control method 1, the success rate was less than $10 \%$. If we were to rely on this method there would only be a very slight chance of success. Control method 2 improved on control method 1, albeit marginally. This resulted in a success rate of only $18.2 \%$ which, depending on the cost of the control, may be inadequate. For control method 3 , if we required a success probability of $95 \%$, this would mean we would have to destroy a very large area over half a million square kilometres.

For the improved scenario, where the improved values of $L o S=0.3$ and $L o D=0.01$ were used, we saw a large increase in the success rate of both paddock control methods and this was also true for control method 3 . The success rate of control method 1 improved from $8.2 \%$ to $58.8 \%$ and control method 2 improved from $18.2 \%$ to $70.9 \%$. The area required to be destroyed for control method 3 to achieve a $95 \%$ probability of success was greatly reduced from $500,000 \mathrm{~km}^{2}$ to less than $23,000 \mathrm{~km}^{2}$.

Our results showed that the level of surveillance $(L o S)$ and the detectability of the disease $(L o D)$ play a key role in determining the success of a control measure. An early detection time is critical for the success rate of all the control methods. We expect the detection time in general to be heavily dependent on the level of inspection and the detectability of the disease. We postulate that the probability of eradication success can be estimated from these two indices ( $L o S$ and $L o D$ ). If a disease is extremely hard

to detect then the likelihood of detection occurring before the disease has reached ineradicable proportions is slight. Also, if the casual inspection level is low then the disease will go undetected for longer and may reach a point where eradication is no longer feasible.

Using these preliminary results, we have identified detectability as a key trait of rain-splashed crop diseases that indicates their eradicability. In future studies we will extend the analysis to explore the independent effects of the level of surveillance $(L O S)$ and the level of detectability $(L o D)$ on the chance of eradication. However, we expect other factors will also have a significant effect. Future work will use the extended model presented in this paper to investigate other factors that may affect the success of the eradication measures considered. The factors we will investigate, apart from the aforementioned dynamics of the parameters for detection, are mainly changes in the cropping arrangement. We expect the availability of susceptible host in the areas outside the cropping region to play a key role in disease eradicability and may also feature as one of 
the general indices indicating eradicability. We also want to investigate changes in the landscape arrangement that may affect the success of the control methods, such as a reduction in available lupin paddocks and changes to the lupin paddock spatial structure, as well as other environmental factors, such as rainfall. The assumption that the control measures in Section 2.4 (once employed) are 100\% successful may not always be realistic - particularly in the verge areas where a high conservation status may exist. This assumption removes the potential for an imperfect eradication scheme and assumes eradication success is only a function of the level of surveillance and the detectability of the disease. Future analysis could include the effects of an imperfect eradication scheme by simulating the probability that there is some residual disease remaining after control has been carried out. This could be done by specifying a probability that control is $100 \%$ successful and then determining the actual success of the control methods stochastically. Each of the effects listed above will be investigated by running an extensive set of stochastic simulations.

\section{ACKNOWLEDGMENTS}

The majority of this work was carried out while the first author was employed at The University of Western Australia in the School of Plant Biology as part of a project supported by the Cooperative Research Centre for National Plant Biosecurity. The authors would like to acknowledge the support of the Australian Government's Cooperative Research Centres Program, and Dr Ian Foster for supplying the hourly weather data used in our simulations. The first author also acknowledges partial financial support from the Australian Space Research Program (ASRP).

\section{REFERENCES}

Adhikari, K. N., Buirchell, B. J., Thomas, G. J., Sweetingham, M. W. and Yang, H. (2009). Identification of anthracnose resistance in Lupinus albus L. and its transfer from landraces to modern cultivars. Crop and Pasture Science 60(5): 472-479.

Australian Bureau of Statistics. Value of agricultural commodities produced, Australia, 2009-10. Retrieved 13/07/11, from $\quad$ http://www.abs.gov.au/AUSSTATS/abs@.nsf/DetailsPage/7503.0200910? OpenDocument.

Brown, J. K. M. and Hovmøller, M. S. (2002). Aerial dispersal of pathogens on the global and continental scales and its impact on plant disease. Science 297(5581): 537-541.

Clements, J., Buirchel, B., Yang, H., Smith, P., Sweetingham, M. and Smith, C. (2005). Lupin. Genetic resources, chromosome engineering, and crop improvement, CRC Press Taylor \& Francis Group, Florida, USA.

Diggle, A. J., Salam, M. U., Thomas, G. J., Yang, H. A., O'Connell, M. and Sweetingham, M. W. (2002). AnthracnoseTracer: A spatiotemporal model for simulating the spread of anthracnose in a lupin field. Phytopathology 92(10): 1110-1121.

Kot, M., Lewis, M. A. and van den Driessche, P. (1996). Dispersal data and the spread of invading organisms. Ecology 77(7): 2027-2042.

Nirenberg, H. I., Feiler, U. and Hagedorn, G. (2002). Description of Colletotrichum lupini comb. nov. in modern terms. Mycologia 94(2): 307-320.

Paulitz, T. C., Atlin, G. and Gray, A. B. (1995). First report of Colletotrichum gloeosporioides on lupine in Canada. Plant Disease 79: 319.

Shivas, R. G., McClements, J. L. and Sweetingham, M. W. (1998). Vegetative compatibility amongst isolates of Colletotrichum causing lupin anthracnose. Australasian Plant Pathology 27(4): 269-273.

Sweetingham, M. W., Cowling, W. A., Buirchell, B. J., Brown, A. G. P. and Shivas, R. G. (1995). Anthracnose of lupins in Western Australia. Australasian Plant Pathology 24: 271.

Sweetingham, M. W. and Shea, G. (1998). Anthracnose - 1997 spread and industry outlook. Crop Updates, DAFWA, Western Australia.

Talhinhas, P., Sreenivasaprasad, S., Neves-Martins, J. and Oliveira, H. (2002). Genetic and morphological characterization of Colletotrichum acutatum causing anthracnose of lupins. Phytopathology 92(9): 986-996.

Thomas, G. J. and Sweetingham, M. W. (2004). Cultivar and environment influence the development of lupin anthracnose caused by Colletotrichum lupini. Australasian Plant Pathology 33(4): 571-577.

van Heemst, H. D. J. (1986). Physiological principles. Modelling agricultural production: weather, soils and crops. H. van Keulen and J. Wolf, PUDOC, Wageningen, the Netherlands: 13-26.

Yang, H. A. and Sweetingham, M. W. (1998). The taxonomy of Colletotrichum isolates associated with lupin anthracnose. Australian Journal of Agricultural Research 49(8): 1213-1224. 